Int. J. Curr. Res. Med. Sci. (2017). 3(9): 1-8

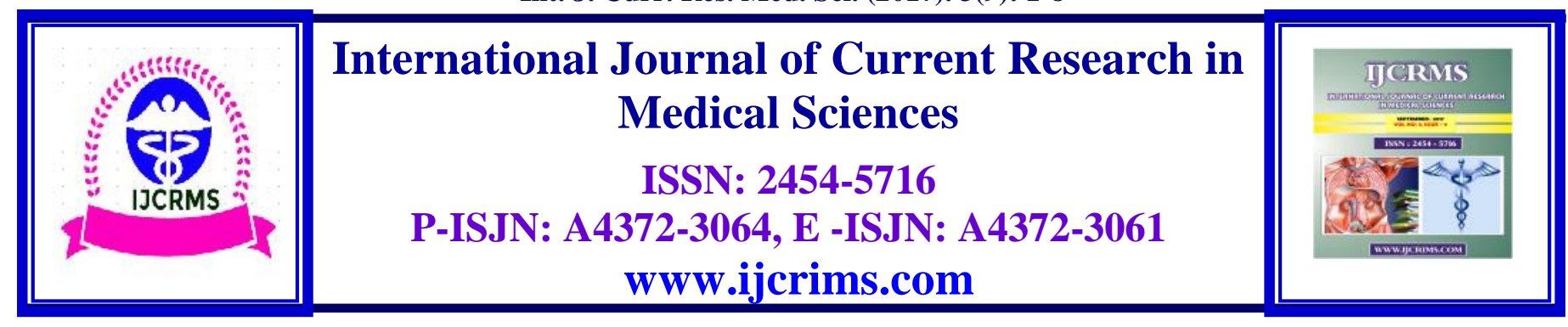

\title{
Comparison of supervised physiotherapy program verses convensional exercises in the management of hemarthrosis in haemophilic patients
}

\author{
Rashmi Maurya ${ }^{1, *}$, Poonam $^{2}$ \\ ${ }^{1}$ Department of Physiotherapy, Assistant Professor, Noida International University, Greater Noida. \\ Uttar Pradesh, India. \\ ${ }^{2}$ Assistant Professor, Department of Physiotherapy, IAMR Ghaziabad, Uttar Pradesh, India. \\ *Corresponding author: rashmimaurya141@gmail.com
}

\begin{abstract}
Hemophilia is a hereditary lifelong blood disease with a problem of blood clotting affecting males. The nature of the condition influence the person to bleed intraarticularly and intermuscularly. Without intervention it can extend to chronic synovitis and severe joint hemarthropathy.

Purpose and Background: Physiotherapeutic interventions are available that may help to prevent and treat the hemophilic patients. Our main objective was to find the most effective physiotherapy procedures for treating haemophilic patients in clinic with respect to conventional therapy at home, In patients of haemophilia with hemarthrosis of knee. The physiotherapy aim was to reduce pain and increase range of flexion and extension of knee and increase the strength of quadriceps muscle

Design: Pre-test post- test comparative study.

Method: 30 haemophilic children's, Taken from the Haemophiliac Federation (India). Males aged between 7-13 years with mean age 10 years having complicated knee hemarthrosis. These children's were randomly divided into two groups A and B of 15 children in each group. Group A- Supervised physiotherapy program performed in the clinic Group B- Conventional Exercises at home. And was compared by repeated measures for 6 months.

Result: The major findings revealed that both the programs were effective in the management of hemophilia but The ROM- flexion, ROM- extension, VAS and the scores of MMT-10 scale improved in subjects who received the supervised physiotherapy program in the clinic than those who received the conventional assisted exercises at home.
\end{abstract}

Keywords: Haemophilia, Heamarthropathy, Bleeds, Knee Joint 


\section{Introduction}

Haemophilia or hemophilia is a Greek name haima means 'blood' and philia means love is a group of hereditary genetic disorders that impair the body's ability to control blood clotting, which is used to stop bleeding when a blood vessel is broken. It is an X linked disorders. It is more likely to occur in males than females ${ }^{1}$. resulting from deficiency of clotting factor VIII (Haemophilia A) or Factor IX (Haemophilia B) ${ }^{2}$ Haemophilia A (clotting factor VIII deficiency) is the most common form of the disorder, present in about 1 in 5,000-10,000 male births.3 Haemophilia B (factor IX deficiency) occurs in around 1 in about 20,000-34,000 male births ${ }^{1}$. affect 1 in 1,000 men ${ }^{3}$ Haemophilia has featured prominently in European royalty and thus is sometimes known as "the royal disease". Queen Victoria passed the mutation to her son Leopold and, to some of her daughters. Like most recessive sex-linked, $\mathrm{X}$ chromosome disorders, Haldane's conclusion that the male mutation rate is much higher than the female rate has stood the test of time for both haemophilia $\mathrm{A}$ and haemophilia $\mathrm{B}{ }^{4}$. Female carriers can inherit the defective gene from either their mother or father, or it may be a new mutation. Only under rare circumstances do females actually have haemophilia.Characteristic symptoms vary with severity. In general symptoms are internal or external bleeding episodes, which are called "bleeds". There are three categories, depending on the percentage of the blood-clotting factor: severe hemophilia ( $<1 \% \mathrm{FVIII} / \mathrm{IX})$, characterised by spontaneous bleeding; moderate hemophilia (1$5 \%$ FVIII/FIX) $^{6}$, with bleeding from slight injuries; and mild hemophilia (>5-40\% FVIII/FIX) with bleeding during surgical procedures The most common musculoskeletal injuries caused by haemophilia are hemarthrosis, synovitis and muscular haematomas. The patient ends up suffering haemophilic arthropathy and functional troubles ${ }^{7}$

With physiotherapy we can achive a lot in patients to relieve their pain sensitivity troubles, stimulating the hemorrhagic reabsorption that are intervening in the inflammatory process and obtaining an appropriate physical condition, improve the life quality and prevent and treat the injuries and their possible consequences. A wide range of techniques can be applied, including physical exercise for patients without injuries, techniques of kinesiotherapy, thermotherapy, electrotherapy, magneto therapy, laser therapy, hydrotherapy and ultrasound therapy are techniques we all use in our daily activities. Each of these techniques have their own specific purpose, ranging from the elimination of the haematoma to the improvement of the mobility.

The use of isometric exercise programs during rehabilitation and maintenance is a promising treatment component for the total care of the individual with haemophilia. ${ }^{16}$ Isometric exercises of the knee contract the muscle fibers, but do not move the angle of the knee, thereby increasing strength and durability for rehabilitation. These exercises are conducted in a static position, rather than being dynamic and utilizing a range of motion. $^{12}$

The patient can start active exercising as soon as possible, and need only stop when restoration is completed, with the aim of preventing muscular atrophy. Contractures of the joints must be avoided and splints or orthoses should be used ${ }^{13}$. Rehabilitation treatment can be achieved through consecutive stages, passing from one to another depending on whether or not there is improvement. Movement is initiated with isometric exercises in such a way that muscular tone is improved and the strength reaches grade 3 (against resistance), removing the immobility, but always under the protective shield of adequate doses of Factor VIII or FIX ${ }^{10}$.

Active resistive exercises are exercises prescribed or performed against progressive resistance to build further muscle strength ${ }^{6}$.Strength training exercises condition the muscles to adapt to new demands and increases strength thus allowing the capability of longer work loads ${ }^{12}$.

Regular exercise may help reduce further destruction in haemophilic joints by strengthening muscle ligaments and tendons surrounding the joint thereby protecting them from damage caused by recurrent haemarthrotic events. Besides these, 
isokinetic exercises like static bicycling can be incorporated later in the patients exercise regime ${ }^{18}$ Gradual walking and stair climbing are also effective in the management of haemophilic patients

\section{Statement question}

Weather the role of physiotherapy is effective in the management of hemarthrosis in haemophilic patients

\section{Need of the study}

Hemarthrosis is the most common, most physically, economically and psychologically debilitating manifestation, occurring in $90 \%$ of affected patients. In fact most of physical, psychological, social and financial problems in haemophilia are caused by the effects of recurrent hemarthrosis, chronic arthropathy, joint deformities and restriction of mobility. The aim of management in haemophilia lies in reducing bleeding frequency and prevention of joint deformities and function

\section{Objectives}

- $\quad$ To reduce the demand of Factor VIII replacement with physiotherapy intervention in the prevention and treatment of recurrent knee hemarthrosis.

- $\quad$ To study the role of physiotherapy with respect to conventional therapy in the management of patients of haemophilia with hemarthrosis of knee

\section{Hypothesis}

\section{Experimental Hypothesis}

There is a significant improvement in the function of knee in supervised physiotherapy program verses conventional exercises in the management of hemarthrosis in haemophilic patients.

Null Hypothesis

There is no significant improvement between supervised physiotherapy program verses conventional exercises in the management of hemarthrosis of knee in haemophilic patients.

\section{Sample and sampling technique}

Randomized sampling According to the financial status of the patient.

Only 30 haemophilic children's, Males aged between 7-13 years with median age of 10 years were selected. All these patients were having complicated knee hemarthrosis. All patients Taken from the Hemophilia Federation (India).

\section{Inclusion Criteria}

- $\quad$ Only haemophilic patients, Males

- $\quad$ Aged 7-13 years with median age of (10) years,

- $\quad$ Complicated with knee hemarthrosis.

- All patients received Factor VIII concentrate at dosage of 30- $40 \mathrm{IU} / \mathrm{kg} \mathrm{I.V}$ daily for 5 days, followed by physiotherapy.

- $\quad$ All patients had history of bleeding episodes into the target joint knee at median frequency of three times per month; their mean ROM at enrollment will be $70^{\circ}$.

\section{Exclusion Criteria}

- Haemophilic hemarthrosis other than knee joint.

- $\quad$ Non haemophilic patients

- $\quad$ Patients had a bleeding history of less than 3 times per month.

\section{Instruments and tools used}

Factor VIII Concentrate: All patients will receive F VIII Concentrate intravenously in a dosage of $30-40 \mathrm{IU} / \mathrm{kg}$ body weight, daily for five days, and then they were referred.

Plinth: Standard 0.9 x $1.8 \mathrm{~m}$ ( 3 x 6feet) plinth used for taking measurements. And treatment Manual Muscle Testing: $0-10$ scale used to measure the muscle strength based on gravity and annually applied resistance. MMT started after 4 weeks of treatment. 
Goniometer: $360^{\circ}$ universal goniometer was used to measure knee angles.

VAS (Visual Analogue Scale): used to measure the intensity of pain.

Ice Pack: Used for ice therapy

Towel Role: 6 inch towel role for exercises.

Wt. Cuffs: 1-kg, 2-kg, 3-kg, Used for active resisted exercises to strengthen muscles.

Static Bicycle: Used for strengthening exercises.

Foot Step: Used for step up and step down exercise

\section{Methodology}

30 subjects with a known cause of moderate to severe haemophilia were taken for the study. Complicated with knee hemarthrosis were selected in the study on basis of the inclusion criteria and divided into two groups of 15 children in each group.

After taking the consent from each individual the patient were randomly assigned into 2 groups of 15 patients each. The patients were then explained about the procedure of the therapeutic process in detail. And each exercise was taught to the patient accurately to avoid bleeding risks specially for the patients who were treated conventionally

Firstly patient is immobilized in a pain free range and cryotherapy is applied for 10 min 2 times a day after 48 hours as pain improves Knee is supported in a maximum extension position and ice therapy is continued for the next 4 weeks. MMT was started after ice therapy treatment i.e. after 4 weeks.

Both the groups were taught Quadriceps Isometrics of knee joint in supine lying position followed with the following exercises for 6 months.

\section{Patients of Group A:}

Active assisted exercises with the help of therapist Active Resisted Exercises with the help of weight cuffs

Stationary bicycle

\section{Patients of Group B:}

Active Assisted Exercises with the help of opposite limp

Active Resisted Exercises with the resistance of opposite limb

Standing on one leg and holding the position

Walking on the edge of a mat

Step up and step down exercise

\section{Data analysis}

Data were summarized as Mean \pm SD. The age of two independent groups Group A \&Group B were compared by Student's t test. The dependent pre and post treatment outcome measures (Flexion, Extension, VAS and MMT-10 scale) of two independent groups were compared by repeated measures analysis of variance (ANOVA) using general linear models (GLM) and the significance of mean difference within and between the groups was done by Newman-Keuls post hoc test. A twotailed $(\alpha=2)$ probability $\mathrm{p}<0.05$ was considered statistically significant. All analyses were performed on SPSS (version 15.0).

\section{Experimental result}

- $\quad$ The pre and post treatment ROM- flexion scores of two groups shows that the mean flexion scores in both the groups increases (improvement) after the treatment and the increase was evident 1.3 times higher in Group A (36.5\%) than Group B (28.4\%). P value was 0.7372 and .0046 respectively.

- The pre and post treatment ROMextension scores of two groups shows that the mean extension scores in both the groups increases (improvement) after the treatment and the increase was evident 1.4 times higher in Group A (26.7\%) than Group B (19.2\%). P value was 0.4237 and 0.002 respectively.

- $\quad$ The pre and post treatment VAS scores of two groups shows that the mean VAS scores in both the groups decreases (improvement) after the treatment and the decrease was evident 1.1 times higher in Group A (91.7\%) than Group B (82.5\%). $\quad \mathrm{P}$ value was 0.6452 and 0.0041 respectively. 
- $\quad$ The pre and post treatment MMT-10 scale scores of two groups shows that the mean MMT10 scale scores in both the groups increases (improvement) after the treatment and the increase was evident 1.2 times higher in Group A $(76.5 \%)$ than Group B (65.2\%). P value was 0.5742 and 0.0001 respectively.

\section{Discussion}

The review of existing literature regarding the role of physiotherapy in hemophilic patients with Hemarthrosis of knee joint, reveals a confusing picture and limited evidence so as to which treatment out of supervised physiotherapy program performed in the clinic verses conventional treatment at home. But none of them have compared the effectiveness of the above two groups. Therefore, the current study was undertaken to investigate and compare the effectiveness among these two groups in improving pain, joint range of motion (ROM) and strength of quadriceps muscle.

For the purpose of this: a two group repeated measure design (experimental study) was carried out. Knee joint was the joint of choice. Since it is the most commonly affected joint in children. The bleeding may often require immobilization of the affected limb to reduce bleeding and pain which leads to loss in quadriceps strength and muscle atrophy $^{20}$. Initially there is loss of extension leading to a knee flexion contracture followed by a posterior subluxation of the tibia with external rotation and valgus deformity ${ }^{21}$.Such patients may become severely handicapped. Sufficient muscular strength is not only necessary for range of movement, but also for absorption of energy. There are a number of studies showing strong positive correlation between knee flexion contracture and leading to joint deformity.

Brenda Buzzard et al 2000: Stated that it is estimated that $80 \%$ of persons with hemophilia world wide do not have access to adequate medical care and only $20 \%$ have access to facilities showing that conventional exercises can be a benefit for these patients ${ }^{19}$.
Exercise should be done several times daily at home for 6 weeks to 3 months combined with icing of the inflamed joint 3-6 times daily for 5-10 $\min ^{22}$.

There are a lot of studies which show that active assisted exercises and active resisted exercises are very effective in the management of hemophilic knee Hemarthrosis. These exercises intend to work on stretching the quadriceps muscle on the anterior front and the hamstrings on the posterior aspect of the leg as well as increase the passive range of motion (ROM) of knee joint flexion and extension. They also focus on developing the quadriceps muscles in a controlled manner. But as the patients are hemophilic a lot of care is required in performing these exercises like these exercises should only be performed within a pain free range of motion subject should feel the tension in the muscle but not pain, which becomes hard to make the subject understand when performing at home and if not taken care leads to frequent bleeding in those subjects. Active assisted exercises and active resisted exercises when performed with the help of patient opposite limb can not be very accurate as these exercises are to be performed in a slow smooth and a controlled motion which can only be taken care by a trained physiotherapist as they can control the motion with their support, but a child might not be that perfect in controlling his movement ${ }^{25}$. Koch et al 1982: Describe a rehabilitation program for individuals with hemophilia ${ }^{23,12}$ they elude in their article to the use of isometric exercise. This program of active resistive exercise begins with a $1-\mathrm{lb}$ weight and may progress to a 4-lb weight. Although the exercise program that they describe may be convenient, it is not vigorous enough to restore full strength to the quadriceps femoris muscles, which may be 10 to 20 times stronger than the resistance allowed for in the study. Individuals are limited to using the weight with which the weakest part of the muscle can complete the $\mathrm{ROM}^{24}$. These exercises should not be done on a soft surface as it will not achieve the desired result which can be done by the therapist but when done at home at times it is possible that the subjects do perform on soft surfaces. All these points made carry the study of 
a comparison between the supervised physiotherapy and the conventional therapy methods.

The study rejected the null hypothesis that no difference would be seen between supervised physiotherapy program verses conventional exercises in the management of hemarthrosis of knee in haemophilic patients. The groups that performed exercises clinically for a period of six months showed a significant greater gain in The ROM- flexion, ROM- extension, VAS and the scores of MMT-10 scale improved 1.3, 1.4, 1.1 and 1.2 fold more respectively in then those who received the conventional assisted exercises at home.

Improvements seen in conventional group was expected with considering the previous research studies regarding the effectiveness of exercises at home.

J S Kale ${ }^{20}$ Conclude that regular exercise regimes may not be followed by all patients on a day-today basis at the hospital clinic due to difficulties of traveling. Training patients and parents for home exercise schedules is important. Many of such patients may not follow the exercise regime at home and often continue to have recurrent bleeding episodes. It may be necessary to have more of community centers offering services to patients staying far off. This indicates a greater number of medical, paramedical and scientific staff to be trained in the field of haemophilia.

There are well documented and reliable methods which are used for documenting the intensity of pain, but because the mean age of this study was 10 years and it was difficult for the subjects to understand the different types of pain only Visual Analogue Scale (VAS) could be used as it was a simple and easy to understand scale. The subjects were asked to point the intensity of pain on a 0 to 10 point scale with no pain to maximum pain. VAS is a reproducible method of expressing pain severity. It takes relatively little time to complete and allows cross culture comparison due to minimal language translations difficulties ${ }^{41}$. Many studies conclude that Visual Analogue Scale is effective measure for measuring pain for the required age group.

The study showed that difference will be seen in the intensity of pain after 6 months of treatment between the supervised physiotherapy and conventional treatment at home. The groups that performed in the clinic under the guidance of physiotherapist showed greater gains in comparison to the subjects with home based exercise.

The measurement of range of motion is an important parameter used in physical therapy evaluation and follow up to measure flexibility for testing the flexion and extension of the knee joint range. The most widely used standardized clinical method of measuring available joint ROM is goniometry. With its units given in degrees ${ }^{14}$. well-trained physical therapists could measure the range of motion of the specified joints with a high degree of reliability and Concluding that "measurements made by different physical therapists may be used interchangeably without destroying the reliability of the clinical records" the subjects were tested in supine lying position and we have selected the active assisted knee flexion and extension and the subjects were asked not to force beyond the point of pain reducing the risk of bleeding.

Khriesat I. Et al ${ }^{26} 2000$ contributed on the fact that there patients present evidence which suggest that physiotherapy program has almost normalized the ROM and decreased bleeding frequency, emphasizing the importance of physiotherapy in treatment of hemophilic knee , being concordant with what was reported by different authors.

Quadriceps atrophy causes inability of the joint, producing repeated bleeding episodes, and hastens the development of serious joint destruction. Based on this many authors advocate, that treatment aims at restoring the original muscle strength as joint stability is dependent on the uniform distribution of muscle power over

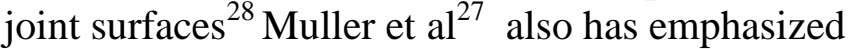
the importance of joint mobilization techniques in advanced hemophilic arthropathy. 
In the present study supervised physiotherapy program showed better gains as compared to conventional therapy at home. Stating that exercises when performed in a controlled manner with the assistance of a trained physiotherapist leads to reduced bleeding causing reduction in pain, improvement in the ROM and muscle strength Although there is a bit gain in the conventional method which is helpful for the patients who do not have facilities, but the family should be taught the effect of a controlled treatment to get better results

\section{Conclusion}

The study compares supervised physiotherapy program performed in the clinic and conventional exercises performed at home. Study found both the programs effective in the management of hemophilia but supervised physiotherapy program performed in the clinic was found to be significantly more effective than conventional exercises performed at home.

The ROM- flexion, ROM- extension, VAS and the scores of MMT-10 scale improved in subjects who received the supervised physiotherapy program in the clinic than at home. To avoid risk of frequent bleeding and complicationof haemophilia like knee joint arthropathy supervised physiotherapy should be preferred.

\section{Future research}

- $\quad$ Future studies are needed to determine if supervised physiotherapy is effective verses conventional therapy at home for other joints such as elbow joint or wrist joint in haemophilic patients.

- Studies can be made supervised physiotherapy versus conventional therapy using orthosis and splints to reduce contracture of the knee joint, in haemophilic patients

- $\quad$ Further studies can be carried out for a sever hemophilic patients with longer duration of treatment, and with a large age group.

\section{References}

1. Deshbhratar, P. (2016). Comparison of Quality of Life in Adults and Children with Haemophilia. Imperial Journal of Interdisciplinary Research, 2(7).

2. Kundu, T., Shaik, T., Singh, P., Sheikh, A., \& Kumat, O. (2015). Management of acute bleeding in severe haemophilia using homeopathic medicines: a multicentric case series. J Homeopath Ayurv Med, 4, 180.

3. Kar, A., Phadnis, S., Dharmarajan, S., \& Nakade, J. (2014). Epidemiology \& social costs of haemophilia in India. The Indian journal of medical research, 140(1), 19

4. Crow, J. F. (2000). The origins, patterns and implications of human spontaneous mutation. Nature Reviews. Genetics, 1(1), 40

5. Goddard, N. J., Rodriguez-Merchan, E. C., \& Wiedel, J. D. (2002). Total knee replacement in haemophilia. Haemophilia, 8(3), 382-386.

6. Cuesta-Barriuso, R., Gómez-Conesa, A., \& López-Pina, J. A. (2013). Physiotherapy treatment in patients with hemophilia and chronic ankle arthropathy: a systematic review. Rehabilitation research and practice, 2013.

7. Physiotherapy treatment in patients with hemophilia and chronic ankle arthropathy: a systematic review. Rehabilitation research and practice, 2013.

8. Christine A. Lee, Erik E.Berntrop, W.Keith Hoots, Textbook of Hemophilia: Weiley Blackwell 2010, 2:36

9. Robert L. Nussbaum, Roderiek R. Meclnness, Thompson and Thompson genetics in medicine: Saunders an imprint of Elsevier 1991, 6: 386-389.

10. Rodriguez-Merchan E.C.Goddard N.J., Musculoskeletal aspects of haemophilia, Blackwell Science Ltd Oxford London (2000).

11. Weers M.E. \& Lowe N.K., A critical review of visual analogue scales in themeasurement of clinical phenomena. Research at Nursing and Health 1990,13: 227-236

12. Pamela K. Levangie, Cynthia C Norkin, Joint structure and function, $4^{\text {th }}$ edition: $414-416$ 
13. L. Heijnen, P. De Kleijn, y for the treatment of articular contractures in haemophilia(2008).

14. E.N. Glick and Margot Lucas, Ice Therapy: Rheumatology, 1969, 10(2): 70-75.

15. Muledr, Kathy, Exercises for patients with haemophilia: World federation of haemophilia 2006:45.

16. Pelletier J.R., Findle T.W., Physical Therapy (1987).

17. Hilberg, T, Herbsle, M., Gabriel, H. H. W., Jeschke, D. and Schramm, W., Proprioception and isometric muscular strength in haemophilic subjects, Haemophilia 2001,7: 582-588.

18. Harris S, Boggio L.N., Exercises may decrease further destruction in the adult haemophilic joint, Haemophilia, 2006, 12:237-240

19. Brenda Buzzard \& Karen Beeton, Physiotherapy Management of Haemophilia, Bleckwell Science Ltd (2000).

20. Hilberg, T, Herbsle, M., Gabriel, H. H. W., Jeschke, D. and Schramm, W., Proprioception and isometric muscular strength in haemophilic subjects, Haemophilia 2001,7: 582-588
21. Ahlberg A, Treatment and Prophylaxis of arthropathy in sevear hemophilia,Clini Orthop 1967; 77:135-146

22. Lily Heijnen \& Muhammad Tariq Sohail, Rehabilitation in Developing Countries, Blackwell Science Ltd 2000:135-140.

23. Koch B, Cohen S, Luban WC, et al: Hemophilia knee rehabilitation techniques, Arch Phys Med Rehabil1982; 63:379-382.

24. Joanne R Pelletier, Thomas W. Findley, and Susan A., Isometric Exercises for an Individual with Hemophilic arthropathy, Haemophilia, 1987, 67:1359-1364

25. Prem P. Gogia, James H. Braatz, Reliability and Validity of Goniometric Measurements at the knee, Phys Ther, 1986, 12 (4):192-195

26. Khriesat I., Thonaibat W., Hammaury M., Haemophiliac Knee: Role of Physiotherapy: Bahrain Medical Bulletin, December 2000, 22:10-14

27. Jules M. Rothstein, Peter J. Miller, Goniometric Reliability in a Clinical Setting, 1983, 63(10):1611-1615

28. Buzzard B.M, Physiotherapy for the prevention of articular contraction in hemophilia. Hemophilia 1999;5:10-15

\begin{tabular}{|c|l|}
\hline \multicolumn{2}{|c|}{ Access this Article in Online } \\
\hline Quick Response Code & Website: \\
& Www.ijcrims.com \\
\hline Subject: \\
\hline
\end{tabular}

How to cite this article:

Rashmi Maurya, Poonam. (2017). Comparison of supervised physiotherapy program verses convensional exercises in the management of hemarthrosis in haemophilic patients. Int. J. Curr. Res. Med. Sci. 3(9): 1-8.

DOI: http://dx.doi.org/10.22192/ijcrms.2017.03.09.001 